\title{
Endovascular Resuscitation with Aortic Balloon Occlusion in Pediatric Trauma
}

\author{
Mitra Sadeghi MD' ${ }^{1}$, David T McGreevy MD' ${ }^{1}$ Rickard Lindgren MD PhD², \\ Kjell Ågren MD² and Tal M Hörer MD PhD ${ }^{1}$ \\ 'Department of Cardiothoracic and Vascular Surgery, Faculty of Medicine and Health, Örebro University Hospital \\ and Örebro University, Örebro, Sweden \\ 2Department of Surgery, Örebro University Hospital, Örebro, Sweden
}

\begin{abstract}
Background: The use of Resuscitative Endovascular Balloon Occlusion of the Aorta (REBOA) in resuscitation and trauma management in adults is increasing. However, there is limited data published concerning its use in pediatric patients.

Methods: We describe a case using REBOA for traumatic hemorrhagic shock in a pediatric patient according to the concept of EndoVascular resuscitation and Trauma Management (EVTM) at Örebro University Hospital in April 2019. Informed consent has been received.

Results: An 11-year-old boy arrived at the emergency room (ER) after a motor vehicle accident. Due to total hemodynamic collapse, cardiopulmonary resuscitation was initiated with return of spontaneous circulation. Zone 1 total REBOA was successfully performed for 7 minutes while damage control surgery was performed and massive transfusion was initiated to stabilize the patient. The patient survived and returned to almost normal daily activity.

Conclusion: REBOA for endovascular resuscitation and trauma management may be an additional method for temporary hemodynamic stabilization in pediatric patients and, in this specific patient, was used instead of resuscitative thoracotomy.
\end{abstract}

Keywords: REBOA; Hemorrhage; Hemorrhagic shock; Endovascular Resuscitation; Pediatric Trauma

\section{INTRODUCTION}

In the pediatric population, blunt polytrauma is a major cause of mortality and morbidity, and abdominal injuries are the third leading cause of death [1]. The anatomy of children differs from that of adult patients making them more exposed to severe internal organ

\section{Corresponding author:}

Dr. Mitra Sadeghi, Department of Cardiothoracic and Vascular Surgery, Faculty of Medicine and Health, Örebro University Hospital and Örebro University, Örebro, Sweden.

Email: mitra.sadeghi@regionoreborlan.se

Author contributions: All authors have contributed to the configuration and writing of the manuscript.

Conflicts of interest: None.

Funding: None.

(c) 2019 CC BY 4.0 - in cooperation with Depts. of Cardiothoracic/ Vascular Surgery, General Surgery and Anesthesia, Örebro University Hospital and Örebro University, Sweden injuries [1]. Immediate aggressive management to control hemorrhagic shock is the key to reducing mortality $[2,3]$. Aortic occlusion in adolescent and pediatric patients has previously shown dismal outcomes, especially in those presenting after blunt trauma with cardiac arrest [4]. Resuscitative endovascular balloon occlusion of the aorta (REBOA) is an endovascular device used for hemorrhage control in adults $[5,6]$. REBOA has primarily been used in the adult population but there is a lack of long-term prospective data. The evidence in the pediatric population is even more limited with only one retrospective observational cohort publication from the Japanese Trauma Data Bank. This includes 54 severely injured children and suggests REBOA as a reasonable option in the pediatric population [7].

To the best of our knowledge, this article presents the first published case of pediatric REBOA used in trauma performed outside Japan and the youngest trauma REBOA survivor known, as well as the youngest survivor after pediatric blunt trauma who presented in cardiac arrest. 


\section{METHODS}

The case data was analyzed using the hospital medical chart. Informed consent was received from the patient's family before writing this article. The Regional Ethics Committee of Uppsala approved the study as part of the ABOTrauma Registry (Study number: 2014/210; EPN Uppsala, Sweden).

\section{Case Presentation}

An 11-year-old boy was brought by air ambulance to Örebro University Hospital after a high-speed motor vehicle collision. He had initially been conscious with stable vital signs, but during helicopter transportation he quickly decompensated with loss of vital signs. $\mathrm{He}$ became unconscious with no palpable pulse or measurable blood pressure and with an arrhythmic heart rate of 45 beats per minute. Impending cardiac arrest led to intubation and the initiation of cardiopulmonary resuscitation and the administration of 1 unit of packed red blood cells (PRBC) and 1 unit of fresh frozen plasma (FFP). Helicopter transportation time was 17 minutes. On arrival to the emergency department (ED), he continued to be pulseless and was noted to have a massive seat belt sign on the abdomen and chest (Figure 1). Signs of cardiac arrhythmic activity were confirmed during the initial FAST (focused assessment with sonography for trauma) assessment as well as detection of hypovolemia and free intraperitoneal fluid.

The patient received an $8 \mathrm{Fr}$ sheath in both the right common femoral artery (CFA) and left common femoral vein using ultrasound guidance. REBOA (ER-REBOA) was inserted and placed uninflated in Zone 1 using landmark guidance while he was being rapidly transported to the operating theater. Total ED time was around 12 minutes. A damage control laparotomy was performed on arrival to the operation room. During laparotomy, the balloon was inflated for 5-7 minutes with $4 \mathrm{ml}$ of saline as invasive systolic blood pressure (SBP) was measured at $40 \mathrm{mmHg}$; it was then used as a bridge to massive transfusion and definitive treatment with a return of spontaneous circulation. As the patient's only other access was intraosseous, the femoral venous access was used for massive transfusion since further peripheral vascular access could not be established due to his hypovolemic state. The balloon was gradually deflated while massive transfusion with 4 units of PRBC, 3 units of FFP and 1 unit of platelets was given as well as fibrinogen and tranexamic acid. The SBP was stabilized at $110 \mathrm{mmHg}$. The injuries consisted of laceration of the abdominal wall and major parts of the intestinal mesentery causing massive hemorrhage from the ileocolic artery and branches, which were ligated. A small retroperitoneal hematoma was also detected. Due to extensive devascularization of the distal parts of the small bowel, 2 meters of the small bowel, including the cecum, was resected

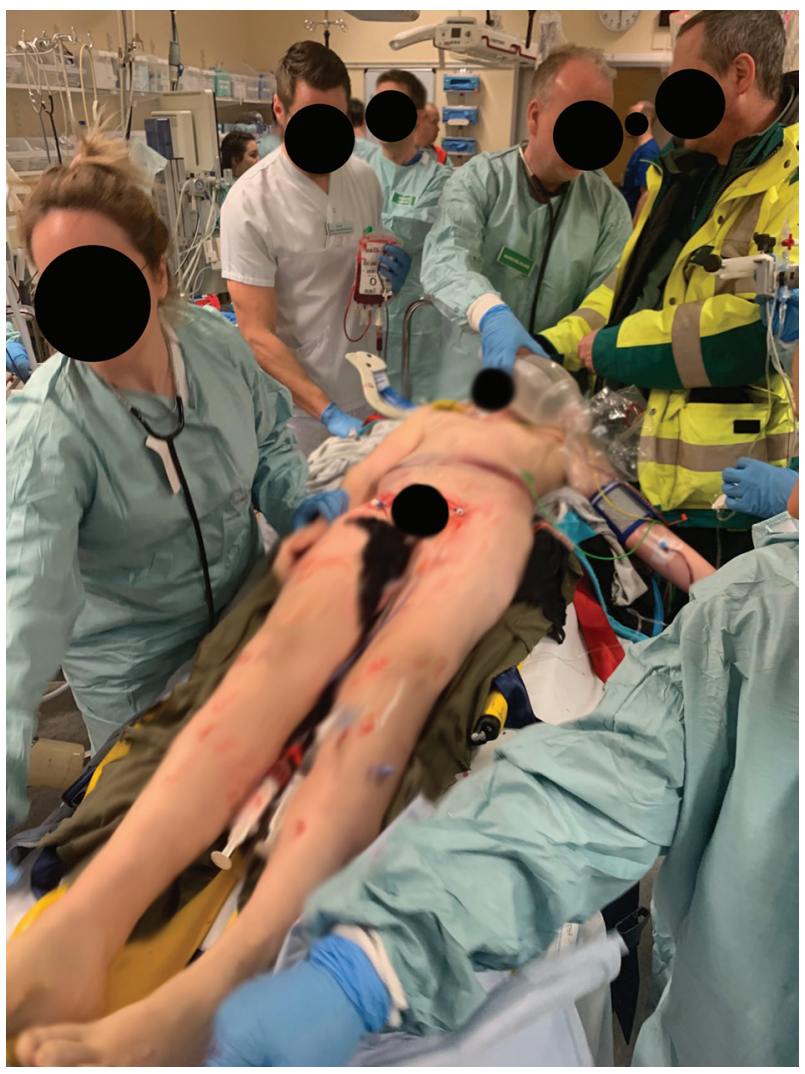

Figure 1 Patient on arrival to the emergency department. Bilateral vascular access and REBOA in situ (Zone I, deflated at this point).

with stapled ends leaving only 1 meter of small bowel remaining. Both introducers were initially removed by manual compression and a femo-stop compression device was placed on the right groin after the surgery was completed. The patient was hemodynamically stable but was kept sedated with an open abdomen. He was subsequently transported for a CT scan which showed a subdural hematoma and subarachnoidal hemorrhage, a craniocervical dissociation, a contrast defect from a vertebral artery, bilateral hemopneumothorax and lung contusions, a right clavicular fracture and costa 3-4 fractures, a thin layer of retroperitoneal hematoma, an unstable L3 chance fracture and a grade 2 aortic injury with intimal laceration at the same level (Figure 2).

The patient was later transported to the intensive care unit (ICU) for continued monitoring and bilateral chest drains were inserted. During second-look surgery, a left diaphragmatic rupture was observed and sutured. Subsequently, an ileocolic anastomosis to the ascending colon was performed, however abdominal wall reconstruction was not possible and the abdomen was closed with a remaining hernia. Regarding the orthopedic damage, fusion of C0-C2 and L2-L4 was performed. The grade 2 aortic injury was treated conservatively with multiple follow-up computed tomography angiography and duplex ultrasound examinations demonstrating a 


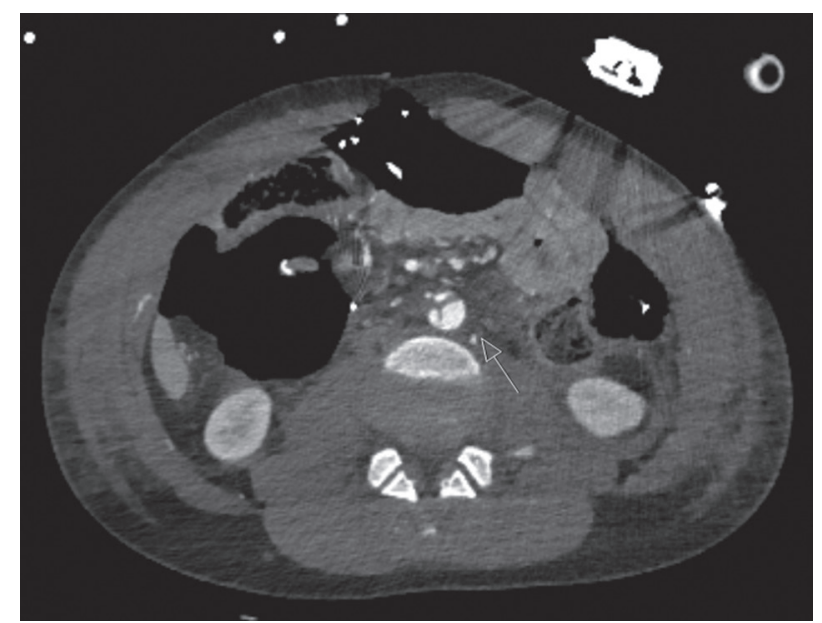

Figure 2 Abdominal CT scan after laparotomy showing a short intimal laceration of the abdominal aorta at the $\mathrm{L} 3$ level.

minor aortic dilation of $15 \mathrm{~mm}$ without progression. As the patient was experiencing neurologic symptoms corresponding to the left brachialis muscle, an MRI was performed showing signs of brachial plexus injury as well as diffuse axonal injury; both were treated conservatively. The patient remained in the ICU for 16 days and was discharged after 48 days. At 90-day follow-up, the patient had only minor paresis remaining in the left arm and has gradually returned to normal activity. Ultrasound did not show further progression of the aortic injury. Abdominal reconstructive surgery is planned.

\section{DISCUSSION}

To the best of our knowledge, this is the first case of pediatric REBOA in trauma performed outside Japan and the youngest trauma REBOA survivor known. This is also the first reported survivor to discharge after pediatric blunt trauma under the age of 14 who presented in cardiac arrest. REBOA was successfully used as a bridge to transfusion and damage control surgery. No complications were reported regarding the femoral accesses.

During the past decade, endovascular trauma management has rapidly evolved. The use of REBOA as an endovascular tool for resuscitating trauma patients with exsanguinating hemorrhage as a bridge to intervention and definitive treatment is increasing worldwide [8-10]. There are still conflicting reports regarding the benefits and dangers of REBOA and randomized studies are lacking [11-14]. The use of REBOA in the pediatric population is even more controversial since only isolated reports are available. Apart from a couple of case reports, there is only one study that has evaluated the efficacy of REBOA in pediatric trauma patients $[7,15,16]$. Previous requirements for using large sheaths (12 Fr) has probably been the reason that REBOA has not been considered in young patients. The alternative, resuscitative thoracotomy (RT) with aortic cross clamping, primarily recommended for penetrating trauma, is rarely performed in this group due to its high mortality rate [17]. In pediatric blunt trauma, there have only been two documented survivors who presented in cardiac arrest and no survivors documented under the age of $14[4,18]$. The introduction of smaller 7 Fr REBOA catheters has now made it technically possible for REBOA to be performed in some of these patients and therefore might be an alternative to RT. However, the question remains regarding if and for whom REBOA may be beneficial.

In this case, REBOA was performed due to traumatic impending cardiac arrest caused by exsanguinating hemorrhage and cardiac arrhythmia according to the EndoVascular Resuscitation and Trauma Management (EVTM) concept $[8,19,20]$. The placement of vascular access in pediatric patients should, however, be carried out with caution as the diameter of the CFA is related to age, body size, and gender. REBOA was placed in Zone 1 to allow control of the life-threatening hemorrhage, as needed, to facilitate rapid transport to the operating theater for definitive treatment. The inflation of the balloon allowed the anesthesiologists to "catch up" on blood transfusion to stabilize the patient and the limited inflation time of 5-7 minutes minimized the risk of visceral and renal ischemia. The amount of saline used for inflation of the REBOA was based on experience using targeted blood pressure level as well as the sensation of resistance when the aorta is totally occluded. It is noteworthy to discuss the cause of the grade 2 aortic injury. In this case, the aortic injury was detected on the CT scan postoperatively and, most probably, was caused by the L3 chance fracture at the same level. However, iatrogenic injury from the REBOA cannot completely be excluded and caution must be taken when inserting the device blindly.

\section{CONCLUSION}

This is the first described pediatric blunt trauma patient presenting in cardiac arrest with no signs of life that survived to discharge under the age of 14 . This landmark patient and description of their care demonstrates that REBOA is a feasible option for the management of pediatric trauma patients presenting in severe hemorrhagic shock. Its use should be considered in the right patient and in the right clinical setting, however further prospective long-term studies in the pediatric patient population are needed.

\section{REFERENCES}

[1] Drexel S, Azarow K, Jafri MA. Abdominal trauma evaluation for the pediatric surgeon. Surg Clin North Am. 2017;97:59-74. 
[2] Spahn DR, Bouillon B, Cerny V, et al. Management of bleeding and coagulopathy following major trauma: an updated European guideline. Crit Care. 2013;17:R76.

[3] Alarhayem AQ, Myers JG, Dent D, et al. Time is the enemy: Mortality in trauma patients with hemorrhage from torso injury occurs long before the "golden hour". Am J Surg. 2016;212:1101-5.

[4] Duron V, Burke RV, Bliss D, Ford HR, Upperman JS. Survival of pediatric blunt trauma patients presenting with no signs of life in the field. J Trauma Acute Care Surg. 2014;77:422-6.

[5] Morrison JJ, Galgon RE, Jansen JO, Cannon JW, Rasmussen TE, Eliason JL. A systematic review of the use of resuscitative endovascular balloon occlusion of the aorta in the management of hemorrhagic shock. J Trauma Acute Care Surg. 2016;80:324-34.

[6] Qasim Z, Brenner M, Menaker J, Scalea T. Resuscitative endovascular balloon occlusion of the aorta. Resuscitation. 2015;96:275-9.

[7] Norii T, Miyata S, Terasaka Y, Guliani S, Lu SW, Crandall C. Resuscitative endovascular balloon occlusion of the aorta in trauma patients in youth. J Trauma Acute Care Surg. 2017;82:915-20.

[8] Hörer T, EVTM group. Top Stent, The Art of EndoVascular Hybrid Trauma and Bleeding Management. Örebro, Sweden: Trio Tryck AB; 2017.

[9] Coccolini F, Stahel PF, Montori G, et al. Pelvic trauma: WSES classification and guidelines. World J Emerg Surg. 2017;12:5.

[10] Sadeghi M, Nilsson KF, Larzon T, et al. The use of aortic balloon occlusion in traumatic shock: first report from the ABO trauma registry. Eur J Trauma Emerg Surg. 2018; 44:491-501.

[11] DuBose JJ, Scalea TM, Brenner M, et al. The AAST Prospective Aortic Occlusion for Resuscitation in Trauma and Acute Care Surgery (AORTA) Registry: Data on contemporary utilization and outcomes of aortic occlusion and resuscitative balloon occlusion of the aorta (REBOA). J Trauma Acute Care Surg. 2016;81:409-19.
[12] Inoue J, Shiraishi A, Yoshiyuki A, Haruta K, Matsui H, Otomo Y. Resuscitative endovascular balloon occlusion of the aorta might be dangerous in patients with severe torso trauma: A propensity score analysis. J Trauma Acute Care Surg. 2016;80:559-66; discussion 66-7.

[13] Norii T, Crandall C, Terasaka Y. Survival of severe blunt trauma patients treated with resuscitative endovascular balloon occlusion of the aorta compared with propensity score-adjusted untreated patients. J Trauma Acute Care Surg. 2015;78:721-8.

[14] Joseph B, Zeeshan M, Sakran JV, et al. Nationwide Analysis of Resuscitative Endovascular Balloon Occlusion of the Aorta in Civilian Trauma. JAMA Surg. 2019;154:500-8.

[15] Hill SJ, Zarroug AE, Ricketts RR, Veeraswamy R. Bedside placement of an aortic occlusion balloon to control a ruptured aorto-esophageal fistula in a small child. Ann Vasc Surg. 2010;24:822 e7-9.

[16] Kobayashi T. Blunt abdominal aortic injury in a child: a case report. JJAAM. 2010;21:343-50.

[17] Moskowitz EE, Burlew CC, Kulungowski AM, Bensard DD. Survival after emergency department thoracotomy in the pediatric trauma population: a review of published data. Pediatr Surg Int. 2018;34:857-60.

[18] Flynn-O'Brien KT, Stewart BT, Fallat ME, et al. Mortality after emergency department thoracotomy for pediatric blunt trauma: Analysis of the National Trauma Data Bank 2007-2012. J Pediatr Surg. 2016;51: 163-7.

[19] Hörer TM, Skoog P, Pirouzram A, Nilsson KF, Larzon T. A small case series of aortic balloon occlusion in trauma: lessons learned from its use in ruptured abdominal aortic aneurysms and a brief review. Eur J Trauma Emerg Surg. 2015;42:585-92.

[20] Hörer T. Resuscitative endovascular balloon occlusion of the aorta (REBOA) and endovascular resuscitation and trauma management (EVTM): a paradigm shift regarding hemodynamic instability. Eur J Trauma Emerg Surg. 2018;44:487-9. 